\title{
Pair Production of Scalar Leptoquarks at the Fermilab Tevatron
}

\author{
M. Krämer, ${ }^{1}$ T. Plehn, ${ }^{2}$ M. Spira, ${ }^{3}$ and P. M. Zerwas ${ }^{2}$ \\ ${ }^{1}$ Rutherford Appleton Laboratory, Chilton, Didcot, OX11 0QX, United Kingdom \\ ${ }^{2}$ Deutsches Elektronen-Synchrotron DESY, D-22603 Hamburg, Germany \\ ${ }^{3}$ TH Division, CERN, CH-1211 Geneva 23, Switzerland
}

(Received 29 April 1997)

\begin{abstract}
We present the cross section for pair production of scalar leptoquarks $L Q$ in $p \bar{p}$ collisions, $p+\bar{p} \rightarrow L Q+\overline{L Q}+X$, at the Fermilab Tevatron in next-to-leading order QCD. Including the higher-order corrections stabilizes the theoretical prediction and increases the size of the cross section for renormalization/factorization scales close to the mass of the leptoquarks. This leads to a rise of the lower bound on the mass of scalar leptoquarks by up to $15 \mathrm{GeV}$ with respect to earlier analyses. [S0031-9007(97)03623-5]
\end{abstract}

PACS numbers: 13.85.Rm, 12.38.Bx, 12.60. $-\mathrm{i}, 14.80 .-\mathrm{j}$

Theoretical speculations have focused on two different elements to explain the recently observed surplus of deepinelastic scattering $e^{+} p$ events at HERA [1]: contact interactions and narrow resonance formation at a mass scale $M \sim 200 \mathrm{GeV}$. The resonance interpretation is based in particular on the $\mathrm{H} 1$ data, which appear to cluster in a narrow range at invariant $(e q)$ masses of about $200 \mathrm{GeV}$. Such resonances [2-6] can be identified with scalar squarks in supersymmetric theories with $R$-parity breaking, or with leptoquarks in general.

The most powerful competitor in this scenario is the pair production of leptoquarks at the Tevatron

$$
p+\bar{p} \rightarrow L Q+\overline{L Q}+X .
$$

Mass bounds on vector leptoquarks, which decay solely into charged leptons and quarks, appear significantly above $200 \mathrm{GeV}$ in the analyses of Refs. [4,5,7]. The present limits for scalar leptoquarks are near $200 \mathrm{GeV}$ [7]. Since they are parameter free, these limits are particularly important: The cross sections for pair production of scalar leptoquarks involve only the strong coupling constant, and they do not depend on unknown Yukawa couplings.

Anticipating the refinement of the Tevatron limits on the masses of scalar leptoquarks in the near future, a solid theoretical prediction of the production cross section [8] is mandatory, the more so as the gap narrows between the Tevatron mass bounds and the HERA mass estimates. Based on previous experience from the production of squark pairs in hadron collisions [9], higher-order QCD corrections are expected to increase the production cross section compared to the predictions at the Born level. Experimental mass bounds are therefore shifted upwards. Moreover, by reducing the dependence of the cross section on spurious parameters, i.e., the renormalization and factorization scales, the cross sections in next-to-leading order (NLO) QCD are under much better theoretical control than the leading-order (LO) estimates. The NLO analysis will be presented in this Letter.

The basic processes for the production of leptoquark pairs at the Tevatron are quark-antiquark annihilation and gluon-gluon fusion:

$$
\begin{aligned}
& q+\bar{q} \rightarrow L Q+\overline{L Q}, \\
& g+g \rightarrow L Q+\overline{L Q} .
\end{aligned}
$$

Any non-point-like structure of leptoquarks that may occur in compositeness scenarios is expected at scales only above $\sim 1 \mathrm{TeV}$; in other scenarios leptoquarks are generically point-like particles. The gluon-leptoquark interactions are therefore determined by the non-Abelian $\mathrm{SU}(3)_{C}$ gauge symmetry of scalar QCD so that the theoretical predictions for the pair production of scalar leptoquarks do not depend on unknown parameters.

The diagrams corresponding to the processes (2), are shown in Fig. 1(a); the only new element of scalar QCD is the quartic coupling between gluons and leptoquarks, which follows from the $\mathrm{SU}(3)_{C}$ gauge invariance of the interaction. The cross sections of the parton processes (2) are given by [10]

$$
\begin{aligned}
\hat{\sigma}_{\mathrm{LO}}[q \bar{q}]= & \frac{\alpha_{s}^{2} \pi}{\hat{s}} \frac{2}{27} \beta^{3}, \\
\hat{\sigma}_{\mathrm{LO}}[g g]= & \frac{\alpha_{s}^{2} \pi}{96 \hat{s}}\left[\beta\left(41-31 \beta^{2}\right)\right. \\
& \left.+\left(18 \beta^{2}-\beta^{4}-17\right) \ln \frac{1+\beta}{1-\beta}\right],
\end{aligned}
$$

(a)
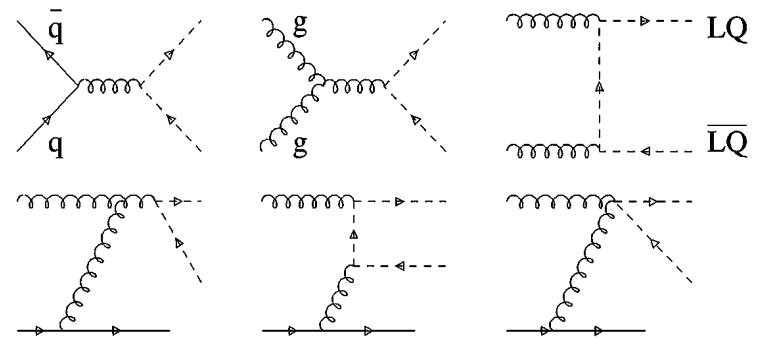

(b)

FIG. 1. Generic diagrams for pair production of scalar leptoquarks in hadron collisions: (a) $q \bar{q}$ annihilation and $g g$ fusion; (b) the gluon-quark subprocess. 
where $\sqrt{\hat{s}}$ is the invariant energy of the subprocess and $\beta=\sqrt{1-4 M_{\mathrm{LQ}}^{2} / \hat{s}}$. The cross sections coincide with those for squark-pair production in the limit of large gluino masses [9]. The quark-antiquark annihilation is the driving mechanism at the Tevatron for leptoquark masses $M_{\mathrm{LQ}} \gtrsim 100 \mathrm{GeV}$.

The QCD radiative corrections to the order $\alpha_{s}$ include virtual corrections, the bremsstrahlung of gluons, and contributions from gluon-quark collisions. The virtual corrections can be classified in self-energy diagrams and vertex corrections for quarks, gluons and leptoquarks, and initial/final-state interactions. In addition to gluon radiation off all colored lines, gluons can also be emitted from scalar vertices. Finally, the inelastic Compton process, diagram (1b), must be added in the order $\alpha_{s}^{3}$ of the cross section. The amplitudes have been evaluated in the Feynman gauge. After the singularities are isolated by means of dimensional regularization, the renormalization is carried out in the modified minimal-subtraction $(\overline{\mathrm{MS}})$ scheme. The masses of the light quarks $(u, d, s, c, b)$ have been neglected while the mass parameter of the leptoquark has been defined on-shell. We have chosen a renormalization and factorization scheme in which the massive particles (top quark, leptoquark) are decoupled smoothly for momenta smaller than their mass [11]. This implies that the heavy particles do not contribute to the evolution of the QCD coupling and the parton densities. The calculation of the cross section for gluon emission has been performed by adopting the phase space slicing method (see, e.g., [12]): A cutoff $\Delta$ is introduced for the invariant mass of the leptoquark-gluon system in the final state, which separates soft from hard gluon radiation. If both contributions are added up, any $\Delta$ dependence disappears from the total cross section for $\Delta \rightarrow 0$. The infrared singularities cancel when the emission of soft gluons is added to the virtual corrections. The remaining collinear initial-state singularities are absorbed into the renormalization of the parton densities [13], defined in the $\overline{\mathrm{MS}}$ factorization scheme.

The perturbative expansion of the total parton cross section can be expressed in terms of scaling functions

$$
\begin{aligned}
& p t \hat{\sigma}_{i j}\left(s, M_{\mathrm{LQ}}^{2}\right) \\
& =\frac{\alpha_{s}^{2}\left(\mu^{2}\right)}{M_{\mathrm{LQ}}^{2}}\left[f_{i j}^{(0)}(\eta)+4 \pi \alpha_{s}\left(\mu^{2}\right)\right. \\
& \left.\quad \times\left\{f_{i j}^{(1)}\left(\eta, r_{t}\right)+\bar{f}_{i j}^{(1)}(\eta) \ln \frac{\mu^{2}}{M_{\mathrm{LQ}}^{2}}\right\}\right],
\end{aligned}
$$

with $i, j=g, q, \bar{q}$ denoting the initial-state partons. For simplicity, we have identified the renormalization scale with the factorization scale $\mu_{R}=\mu_{F}=\mu$. The scaling functions depend on the invariant parton energy $\sqrt{\hat{s}}$ through $\eta=\hat{s} / 4 M_{\mathrm{LQ}}^{2}-1$ and, very mildly, on the ratio of the particle masses $r_{t}=m_{\mathrm{top}} / M_{\mathrm{LQ}}$. The scaling functions $f_{i j}^{(0,1)}$ and $\bar{f}_{i j}^{(1)}$ are displayed in Fig. 2 for the quark-antiquark, gluon-gluon, and gluon-quark channels. The scaling functions $f_{i j}^{(1)}$ are decomposed into a

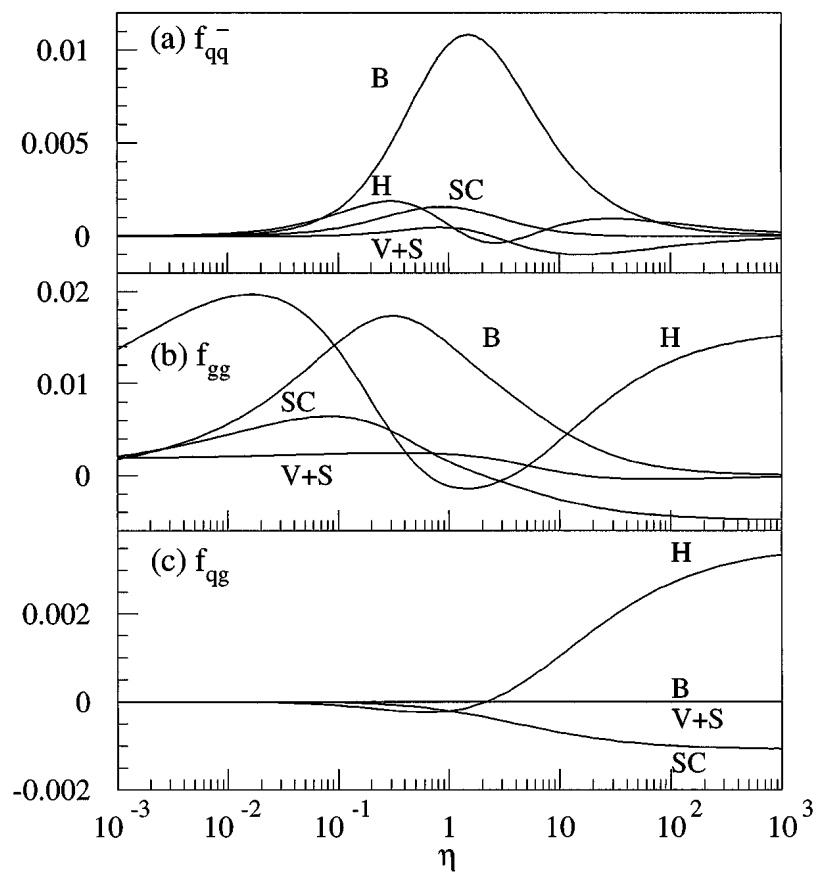

FIG. 2. The scaling functions for pair production of scalar leptoquarks in $q \bar{q}(\mathrm{a}), g g(\mathrm{~b})$, and $g q$ (c) collisions, versus $\eta=\hat{s} / 4 M_{\mathrm{LQ}}^{2}-1$. The notation follows Eq. (4); $B$ denotes the lowest-order scaling function, $V+S$ the sum of virtual and soft corrections, $H$ the contribution of hard-gluon emission, SC the scaling function $\bar{f}$.

"virtual + soft" $(V+S)$ part, and a "hard" $(H)$ gluonradiation part; the $\ln ^{j} \Delta(j=1,2)$ singularities of the $(V+S)$ cross section are mapped into $(H)$, canceling the logarithms in $(H)$ so that all scaling functions are independent of $\Delta$ in the limit $\Delta \rightarrow 0$.

From Fig. 2(b) we can infer that the NLO corrections to the gluon-gluon channel are very important near the threshold $\sqrt{\hat{s}} \geqslant 2 M_{\mathrm{LQ}}$. The large size of the corrections close to the threshold is partly due to gluon radiation off the initial-state partons, which generates contributions of the type $\beta \ln ^{j} \beta(j=1,2)$; the $\ln ^{2} \beta$ terms are universal and can be exponentiated. At the threshold $\sqrt{\hat{s}} \rightarrow 2 M_{\mathrm{LQ}}$, the NLO cross section for the $g g$ initial states is nonzero: The Sommerfeld rescattering correction, due to the exchange of Coulomb gluons between the leptoquark pair in the final state, gives rise to a $1 / \beta$ singularity, which compensates the phase space factor $\beta$. At large parton energies, the hard coefficients $f_{g g}^{(1, H)}$ and $f_{g q}^{(1)}$ approach plateaus, which are built up by the flavor-excitation and gluon-splitting mechanisms. The exchange of gluons in the $t$ and $u$ channels leads to an asymptotically constant cross section, to be contrasted with the scaling behavior $\sim 1 / \hat{s}$ of the $\mathrm{LO}$ process.

The $p \bar{p}$ cross section is found by folding the parton cross sections with the gluon and light-quark luminosities in $p \bar{p}$ collisions. For the numerical analysis we have 


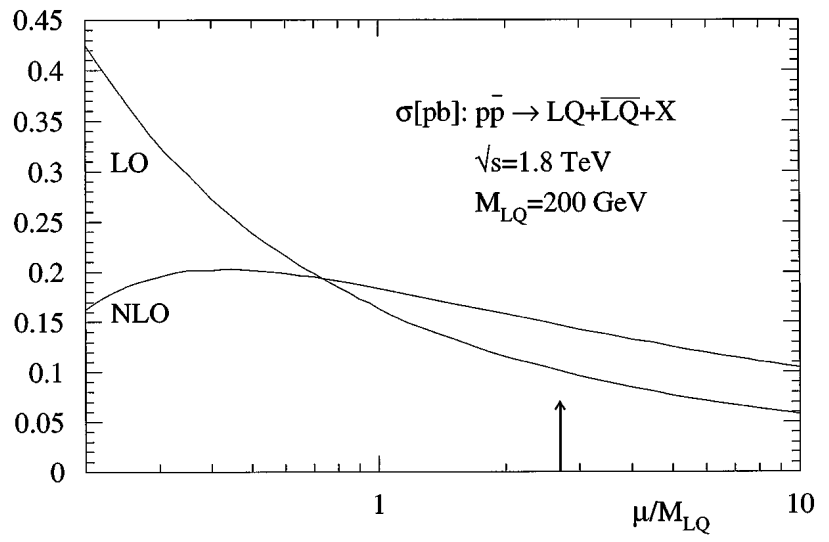

FIG. 3. Renormalization/factorization scale dependence of the cross section $\sigma(p+\bar{p} \rightarrow L Q+\overline{L Q}+X)$ at the Tevatron energy $\sqrt{s}=1.8 \mathrm{TeV}$. Parameters as described in the text. The arrow indicates the average invariant energy $\langle\hat{s}\rangle^{1 / 2}$ in the hard subprocess.

adopted the CTEQ4M parametrization of the parton densities [14]. The QCD coupling is evaluated in the $\overline{\mathrm{MS}}$ scheme for $n_{l f}=5$ active flavors and $\Lambda^{(5)}=202 \mathrm{MeV}$, and the top quark mass is set to $m_{\text {top }}=175 \mathrm{GeV}$ [15].

The scale dependence of the theoretical prediction is significantly reduced when higher-order QCD corrections are included. This is demonstrated in Fig. 3, in which we compare the renormalization/factorization scale dependence at the LO and NLO of the total cross section. For a consistent comparison of the LO and NLO results, we have calculated all quantities [i.e., $\alpha_{s}\left(\mu^{2}\right)$, the parton densities, and the partonic cross sections] in LO and NLO. The scale dependence of the LO cross section is steep and monotonic: Changing the scale from $\mu=2 M_{\mathrm{LQ}}$ to $\mu=M_{\mathrm{LQ}} / 2$ increases the $\mathrm{LO}$ cross section by $100 \%$. At NLO the scale dependence is strongly reduced to about $30 \%$ in this interval. The NLO cross section runs through a broad maximum near $\mu \sim M_{\mathrm{LQ}} / 2$, which supports the stable behavior in $\mu$.

TABLE I. Results for the cross section $p+\bar{p} \rightarrow L Q+$ $\overline{L Q}+X$ at the Tevatron energy $\sqrt{s}=1.8 \mathrm{TeV}$ for various values of the leptoquark mass $M_{\mathrm{LQ}}$ in LO and NLO. Also shown is the $K$ factor defined as $K=\sigma_{\mathrm{NLO}} / \sigma_{\mathrm{LO}}$. Parameters as described in the text. (The negative sign of $\Delta \sigma_{g q}$ is an artifact of subtracting collinear initial-state singularities via mass factorization.)

\begin{tabular}{clccccc}
\hline \hline$M_{\mathrm{LQ}}(\mathrm{GeV})$ & & $\sigma_{q \bar{q}}$ & $\sigma_{g g}$ & $\Delta \sigma_{g q}$ & $\sigma_{\text {tot }}(\mathrm{pb})$ & $K$ \\
\hline \multirow{2}{*}{150} & LO & 0.741 & 0.244 & & 0.985 & \\
& NLO & 0.722 & 0.490 & -0.028 & 1.184 & 1.20 \\
175 & LO & 0.318 & 0.071 & & 0.389 & \\
& NLO & 0.311 & 0.146 & -0.010 & 0.447 & 1.15 \\
200 & LO & 0.142 & 0.022 & & 0.164 & \\
& NLO & 0.141 & 0.047 & -0.004 & 0.184 & 1.12 \\
250 & LO & 0.030 & 0.003 & & 0.033 & \\
& NLO & 0.030 & 0.006 & -0.001 & 0.035 & 1.08 \\
\hline \hline
\end{tabular}

The QCD radiative corrections enhance the cross section for the production of leptoquarks above the central value $\mu \sim M_{\mathrm{LQ}}$. If the $\mathrm{LO}$ cross section is calculated at large scales $\mu \sim \sqrt{\hat{s}}$, the enhancement in NLO is as large as 70\%, nearly independent of the leptoquark mass. The convergence of the perturbative approach should, however, be judged by examining a properly defined $K$ factor, $K=\sigma_{\mathrm{NLO}} / \sigma_{\mathrm{LO}}$, with all quantities in the numerator and denominator calculated consistently in NLO and LO, and evaluated at the central scale $\mu=M_{\mathrm{LQ}}$. In the interesting mass range between $150 \leq M_{\mathrm{LQ}} \leq 250 \mathrm{GeV}$, these $K$ factors vary only between 1.20 and 1.08 , as shown in Table I. They are small enough to ensure a reliable perturbative expansion [16].

The impact of the NLO QCD corrections on the present experimental lower mass limits for scalar leptoquarks is illustrated in Fig. 4(a). We compare the NLO result based on the default settings (CTEQ4M parton densities
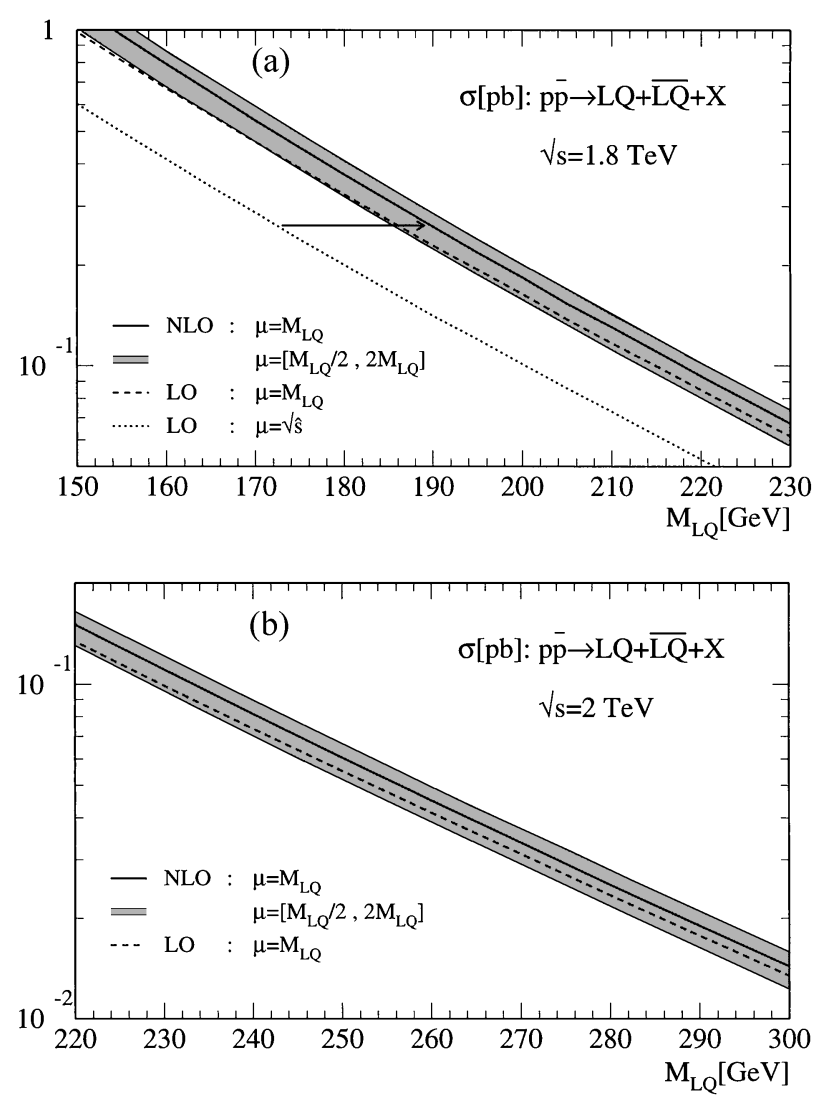

FIG. 4. (a) The cross section for the production of scalar leptoquark pairs, $p+\bar{p} \rightarrow L Q+\overline{L Q}+X$, at the Tevatron energy $\sqrt{s}=1.8 \mathrm{TeV}$ as a function of the leptoquark mass $M_{\mathrm{LQ}}$. The NLO result is compared with LO calculations. The variation of the NLO cross section with the value of the renormalization/factorization scale is indicated by the shaded band. The increase of the lower bound on the leptoquark mass compared to earlier analyses, is demonstrated by the horizontal arrow. (b) The prediction is shown for the cross section at the future Tevatron energy $\sqrt{s}=2 \mathrm{TeV}$ in NLO [signatures as in (a)]. 
and $\left.\mu=M_{\mathrm{LQ}}\right)$ with the LO cross section at the scale $\mu=\sqrt{\hat{s}}$ [17], adopted in earlier analyses. Taken at face value, the NLO order corrections increase the mass limit for a first-generation scalar leptoquark by about $15 \mathrm{GeV}$. [The shift is much smaller if the LO cross sections are evaluated at the renormalization/factorization scale $\mu=M_{\mathrm{LQ}}$, as demonstrated by the broken line in Fig. 4(a).] The shaded band reflects the remaining theoretical uncertainty at NLO due to the choice of the renormalization/factorization scale when $\mu$ is varied in the range $M_{\mathrm{LQ}} / 2 \leq \mu \leq 2 M_{\mathrm{LQ}}$. Since the cross section in the interesting mass region $M_{\mathrm{LQ}} \gtrsim 150 \mathrm{GeV}$ is built up mainly by the quark-antiquark channels, thus based on well-measured parton densities, the variation between different parton parametrizations is less than $5 \%$.

Evaluated on the basis of the NLO cross sections presented in this Letter, the data from the D0 Collaboration at the Fermilab Tevatron, which were presented in Refs. [7], lead to a parameter-free lower limit of about $M_{\mathrm{LQ}}>$ $190 \mathrm{GeV}$ for scalar leptoquarks decaying to charged leptons. The prediction for the cross section at the energy $\sqrt{s}=2 \mathrm{TeV}$ is shown in Fig. 4(b). Anticipating an increase of the integrated luminosity by more than an order of magnitude, the discovery limit for leptoquarks decaying to charged leptons will be raised to a value well above $300 \mathrm{GeV}$.

We have benefited from discussions and communications with J. Collins, S. Hagopian, S. Lammel, G. Landsberg, H. Montgomery, and T. Rizzo. Special thanks go to J. Blümlein, H. Dreiner, M. Mangano, and $\mathrm{H}$. Weerts for valuable comments on the manuscript.

[1] H1 Collaboration, C. Adloff et al., Z. Phys. C 74, 191 (1997); Zeus Collaboration, J. Breitweg et al., Z. Phys. C 74, 207 (1997).

[2] G. Altarelli, J. Ellis, G.F. Giudice, S. Lola, and M.L. Mangano, Report No. CERN-TH/97-40 (hep-ph/ 9703276); J. Kalinowski, R. Rückl, H. Spiesberger, and P.M. Zerwas [Z. Phys. C (to be published)]; J.L. Hewett and T. G. Rizzo, Report No. SLAC-PUB-7430 (hep-ph/ 9703337).

[3] D. Choudhury and S. Raychaudhuri, Report No. CERNTH/97-026 (hep-ph/9702392); H. Dreiner and P. Morawitz, Report No. RAL (hep-ph/9703279); T. Kon and T. Kobayashi, Report No. ITP-SU-97/02 (hep-ph/ 9704221); R. Barbieri, A. Strumia, and Z. Berezhiani, Report No. IFUP-YH 13/97 (hep-ph/9704275).

[4] K. S. Babu, C. Kolda, J. March-Russell, and F. Wilczek, Report No. IASSNS-HEP-97-04 (hep-ph/9703299).

[5] J. Blümlein, Report No. DESY 97-032 (hep-ph/9703287) [Z. Phys. C (to be published)].
[6] Z. Kunszt and W. J. Stirling, Report No. DTP/97/16 (hepph/9703427) [Z. Phys. C (to be published)]; T. Plehn, H. Spiesberger, M. Spira, and P.M. Zerwas, Report No. DESY 97-043 (hep-ph/9703433) [Z. Phys. C (to be published)]; C. Friberg, E. Norrbin, and T. Sjöstrand, Report No. LU-TP- 97-04 (hep-ph/9704214).

[7] D0 Collaboration, J. Wrightman et al., in Proceedings of les Rencontres de la Vallee d'Aoste, 1997 (to be published); D0 Collaboration, J. Hobbs et al., in Proceedings of the XXXII Rencontres de Moriond, 1997 (to be published).

[8] Soft gluon corrections to the production of leptoquark pairs were discussed in M. de Montigny and L. Marleau, Phys. Rev. D 41, 3523 (1990) [based, though, on erroneous Born calculations].

[9] W. Beenakker, R. Höpker, M. Spira, and P. M. Zerwas, Phys. Rev. Lett. 74, 2905 (1995); Nucl. Phys. B492, 51 (1997).

[10] J. A. Grifols and A. Méndez, Phys. Rev. D 26, 324 (1982); I. Antoniadis, L. Baulieu, and F. Delduc, Z. Phys. C 23, 119 (1984); E. Eichten, I. Hinchliffe, K. D. Kane, and C. Quigg, Rev. Mod. Phys. C 56, 579 (1984); G. Altarelli and R. Rückl, Phys. Lett. 144B, 126 (1984); S. Dawson, E. Eichten, and C. Quigg, Phys. Rev. D 31, 1581 (1985); J. Bümlein, E. Boos, and A. Kryukov, Report No. DESY 96-174 (hep-ph/9610408) [Z. Phys. C (to be published)].

[11] J. Collins, F. Wilczek, and A. Zee, Phys. Rev. D 18, 242 (1978); W. J. Marciano, Phys. Rev. D 29, 580 (1984); P. Nason, S. Dawson, and R. K. Ellis, Nucl. Phys. B303, 607 (1988).

[12] W. Beenakker, H. Kuijf, W. L. van Neerven, and J. Smith, Phys. Rev. D 40, 54 (1989).

[13] G. Altarelli, R. K. Ellis, and G. Martinelli, Nucl. Phys. B157, 461 (1979); W. Furmanski, and R. Petronzio, Z. Phys. C 11, 293 (1982).

[14] H. L. Lai et al., Phys. Rev. D 55, 1280 (1997).

[15] CDF Collaboration, F. Abe et al., Phys. Rev. Lett. 74, 2626 (1995); D0 Collaboration, S. Abachi et al., Phys. Rev. Lett. 74, 2632 (1995).

[16] The results nearly coincide with the cross sections for the production of squark-antisquark pairs in the limit of large gluino mass. Supersymmetry predicts quartic self-interactions to order $\alpha_{s}^{2}$ between the squarks. No such self-couplings have been introduced for leptoquarks. However, these couplings affect the cross sections only through rescattering corrections involving heavy leptoquark loops so that their impact on the cross section is small in the weak-coupling scenario.

[17] It is not legitimate to use $\mu=\sqrt{\hat{s}}$ beyond LO since this choice of scale results in an error of order $\alpha_{s}$, no matter how accurately the hard-scattering cross section is calculated [see, e.g., J. Collins, D.E. Soper, and G. Sterman, in Perturbative Quantum Chromodynamics, edited by A.H. Mueller (World Scientific, Singapore, 1989)]. By contrast, the choice $\mu=M_{\mathrm{LQ}}$ is legitimate and, moreover, provides a reasonable starting point for the perturbative expansion by leading to well-controlled higher-order corrections as evident from Fig. 3. 